\title{
Congruence between patients' preferred and perceived participation in medical decision-making: a review of the literature
}

\author{
Linda Brom ${ }^{1 *}$, Wendy Hopmans ${ }^{1}$, H Roeline W Pasman ${ }^{1}$, Danielle RM Timmermans ${ }^{1}$, Guy AM Widdershoven ${ }^{2}$ \\ and Bregje D Onwuteaka-Philipsen ${ }^{1}$
}

\begin{abstract}
Background: Patients are increasingly expected and asked to be involved in health care decisions. In this decision-making process, preferences for participation are important. In this systematic review we aim to provide an overview the literature related to the congruence between patients' preferences and their perceived participation in medical decision-making. We also explore the direction of mismatched and outline factors associated with congruence.

Methods: A systematic review was performed on patient participation in medical decision-making. Medline, PsycINFO, CINAHL, EMBASE and the Cochrane Library databases up to September 2012, were searched and all studies were rigorously critically appraised. In total 44 papers were included, they sampled contained 52 different patient samples.

Results: Mean of congruence between preference for and perceived participation in decision-making was 60\% (49 and 70 representing $25^{\text {th }}$ and $75^{\text {th }}$ percentiles). If no congruence was found, of 36 patient samples most patients preferred more involvement and of 9 patient samples most patients preferred less involvement. Factors associated with preferences the most investigated were age and educational level. Younger patients preferred more often an active or shared role as did higher educated patients.

Conclusion: This review suggests that a similar approach to all patients is not likely to meet patients' wishes, since preferences for participation vary among patients. Health care professionals should be sensitive to patients individual preferences and communicate about patients' participation wishes on a regular basis during their illness trajectory.
\end{abstract}

Keywords: Patient participation, Decision-making, Patient involvement, Congruence, Review

\section{Background}

Making medical treatment decisions can be difficult for patients and physicians. Both patients and physicians are increasingly expected to possess communication skills that facilitate patient participation [1,2]. It has also been argued that patients often need to be more assertive and involved to enable a patient-centred approach to medical decision-making $[3,4]$. In order to place the patient at the centre of care, it is expected that physicians perform their role in a less authoritarian manner [5]. A survey among physicians from different medical disciplines

\footnotetext{
* Correspondence: eol@vumc.nl

'Department of Public and Occupational Health, EMGO Institute for Health and care research, VU University Medical Center, Van der Boechorststraat 7, 1081 Amsterdam, BT, The Netherlands

Full list of author information is available at the end of the article
}

showed that they generally tended to be quite open towards patient participation [6]. However, research among patients shows that the majority do not always feel that their level of participation in medical decisionmaking is sufficient [7].

Preferences for participation in medical decisionmaking are often measured with tools such as the Control Preferences Scale (CPS) [8], evidence suggest the CPS is clinically relevant, easily administered and valid in health care decision-making [8]. The scale has been used in a variety of populations and settings, and administered in several ways, including written, verbal, using answering cards, or using only a 5-point scale.

Two systematic reviews addressed the issue of preferences for participation in medical decision-making. Chewning et al. [9] included various patient populations 
and reported a preference for shared or active decisionmaking, and noted that this preference for participation increased over the past three decades. Tariman et al. [10] reviewed studies on patients with different types of cancer that compared their preferred and perceived role during decision-making. All studies in this review showed discrepancies between preferred and perceived roles in decision-making, and in the majority of the studies patients preferred to be more involved in decision-making than perceived.

Additionally, several original studies have reported on factors associated with preferred role in medical decisionmaking. Studies have shown that younger patients, higher educated people and women more frequently prefer a more active role in decision-making [11,12]. In contrast, older people were found to more often favour a more 'paternalistic attitude' from their physician [13]. The phase in the illness trajectory also appears to influence patient preferences regarding participation. For example, a study among patients with prostate cancer showed that they preferred a more active role later in their disease, possibly because of getting used to being ill [14].

We performed a review including any study population with a strong focus on the congruence between a patient's preferred and perceived role in decision-making. We also investigated factors associated with preferences, perceived participation and the congruence between these two. Of importance when exploring this issue, we distinguished between prospective and retrospective measurement of preferences. In some studies preferences are assessed prospectively, that is before decision-making takes place, while in other studies the patient preferences are measured retrospectively, that is after decision-making took place. When measured retrospectively patients probably take into account their evaluation of the decision-making when expressing their preference after the decisionmaking took place, while prospectively measured preferences are not biased by knowledge of the actual decision-making process. However, prospective measures cannot provide insight in changes in preferences that might occur during the decision-making process or over time $[14,15]$.

The aim of our study was to give an overview of the results from studies in which congruence between patients' preferred and their perceived participation in medical decision-making is assessed and when no congruence was measured, if patients preferred more or less participation than perceived. We distinguished prospective and retrospective studies and also extracted data on factors associated with congruence. Knowing more about presence of congruence and (direction of) mismatch in medical decision making is relevant for practice, since a lack of knowledge may result in sub optimal care and serve as a barrier to patient-centred care.

\section{Methods}

\section{Search strategy}

A systematic review of the research literature was carried out to identify studies that examined the congruence between preferred and perceived participation in medical decision-making among patients. To identify relevant studies, we searched Medline, PsycINFO, CINAHL, EMBASE and the Cochrane Library databases up to September 2012. A "medical information specialist" from the Medical Library VU University medical center assisted with development and pilot testing of the search strategy. The following search terms were used, preference, patient participation and decision-making.

\section{Selection criteria}

All inclusion and exclusion criteria were established before conducting the database searches. Studies were included if they met the following criteria: (a) reported patients' preferences and/or patients' perceived participation and the congruence between preferred and perceived patient participation (or this could be calculated from the presented data), (b) used a 3- or 5-point scale measuring involvement of patient participation in decision-making (active, shared, passive) (not necessarily the CPS specifically) and (c) concerned medical treatment decisionmaking. All study types could be included.

Studies were excluded when they concerned preferences for participation in medical decision-making as a surrogate (e.g. in children) and when the manuscript was not written in English.

\section{Study identification and data extraction}

Articles were entered into Reference Manager 11. Titles and abstracts were initially screened by two reviewers independently ( $\mathrm{LB}$ and $\mathrm{WH}$ ) to decide if the full text articles should be obtained. When there was disagreement, this was resolved through discussion with a third reviewer (HRWP). Full text articles were excluded if a more detailed examination showed that papers did not fit the inclusion criteria.

Two pairs of reviewers (LB and BDOP, WH and HRWP) determined final inclusion or exclusion using a standardized checklist of items. We created a checklist including an inventory of the features critical to inclusion and assessment of the study: 1) type of illness, 2) type of treatment decision, 3) prospective or retrospective measurement of preferences, 4) percentage of preferred and/or perceived participation, 5) percentage of congruence and direction of mismatch (preferred more, or preferred less participation than perceived) and 6) associations with socio-demographic factors. We piloted this checklist in four articles for completeness and reliability reviewers. This pilot resulted in no major additions or modification to items in the original checklist. When analysing the articles with the 
checklist, discrepancies between the two reviewers were resolved by consensus and if necessary consultation with a third reviewer.

Patients' preferred and/or perceived participation in the included studies were described in three categories: active, shared or passive. If data was presented on a 5-point scale $(A, B, C, D, E)$, we rearranged it into a 3-point scale $(\mathrm{AB}, \mathrm{C}, \mathrm{DE})$ to create uniformity. A large number of studies measured on a 3-point scale (e.g., mainly the patient, patient and provider equally, mainly the provider) [12,15-22]. Congruence, mismatch and its direction were reported by using data presented in the original article (based on a 3-point scale) or calculated based on the numbers reported for preferred and perceived participation. Congruence was calculated then by counting the number of participants whose preferred and perceived participation was equal and divided it by the total number of participants. Direction of mismatch was calculated by counting number of patients whose preferred participation was different from perceived participation (preferred more, or preferred less participation than perceived) divided it by the total number of participants.

This resulted in three categories, namely: 1) congruent, 2) preferred more participation than perceived or 3) preferred less participation than perceived.

If more than one study sample was described in an included article, data was extracted for the reported samples separately, for example the different groups in randomised controlled trials or when two patient samples were compared within an included article.

We divided the studies and reported the findings separately for preferences and congruence with perceived participation as follows a) prospective vs retrospective studies, b) patients with cancer vs patients without cancer (non-cancer), and c) treatment options asked in general vs specific treatment options.

The Mixed Methods Appraisal Tool (MMAT) was used to appraise the methodological quality of the included studies [23]. The MMAT has been designed for a systematic mixed studies review. The scores range from 0 (no criteria met) to 4 (all criteria met).

\section{Results}

\section{Study selection}

The results of the search strategy are shown in Figure 1. A total of 4299 unique citations were identified. The abstracts were reviewed and 888 articles were selected for a full-text review. The most important reason for exclusion on the basis of the abstract was that studies did not report patient participation in a medical decisionmaking situation, most evaluated outcomes of treatments. A further included 844 studies were excluded after fulltext review as they did not report on congruence between patients' preferences and perceived participation. A total

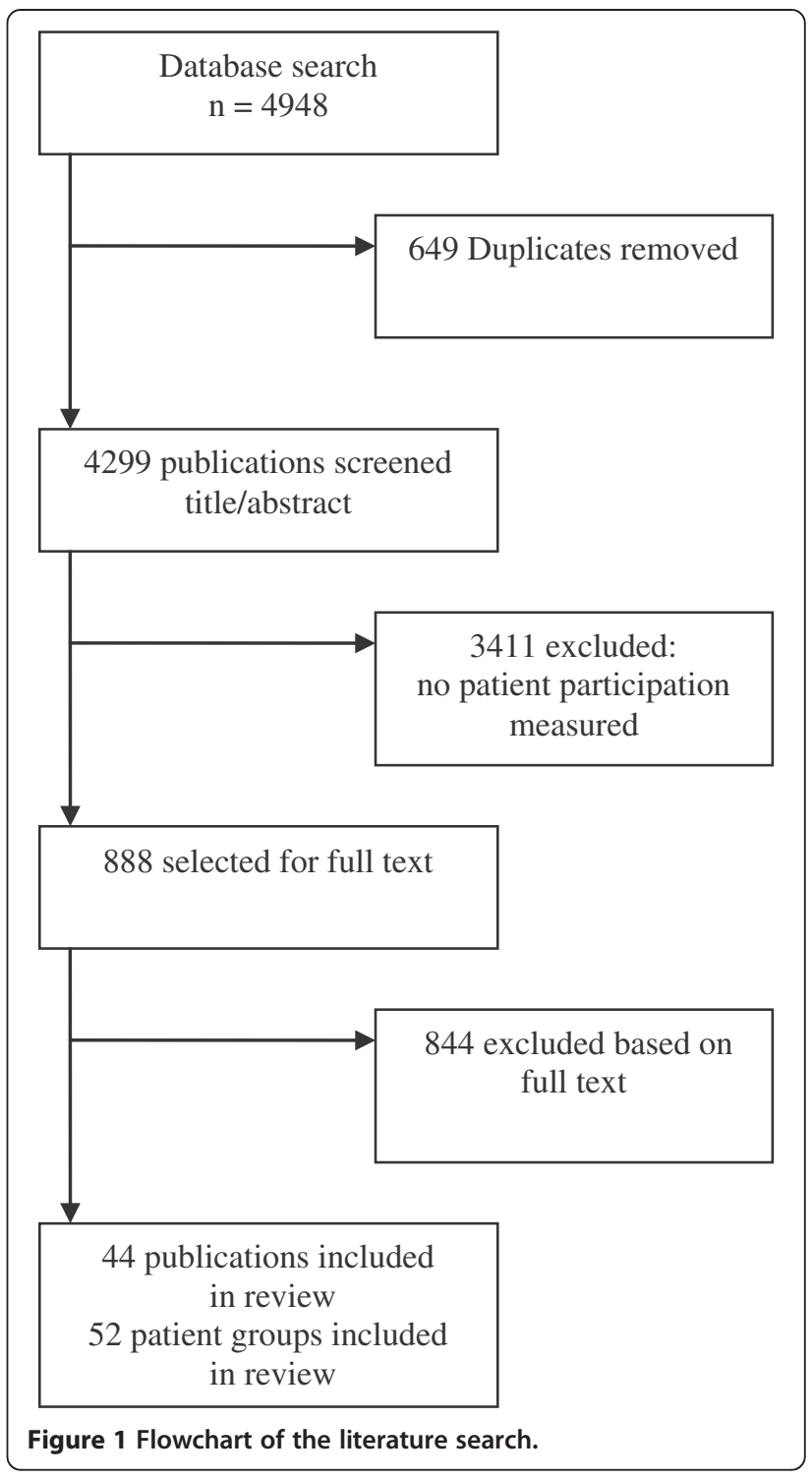

of 44 papers were included which contained 52 different patient samples: A total of 12 studies in which preference was measured prospectively (Table 1 ) reported on 15 patient samples ( 3 RCTs with intervention and control groups). Thirty-two studies in which preference was measured retrospectively (Table 2) were included. One RCT with an intervention group and a control group, and 2 studies comparing patient populations with different types of cancer, resulted in a total of 37 different patient samples.

\section{Study characteristics}

Characteristics of the 12 prospective studies (15 patient samples) are shown in Table 3. A total of 3416 women and 645 men were included in these studies. Mean age was 56.3 years with a range from 21 to 89 years old. 
Table 1 Patients' preferences, congruence and direction of mismatch in medical decision-making

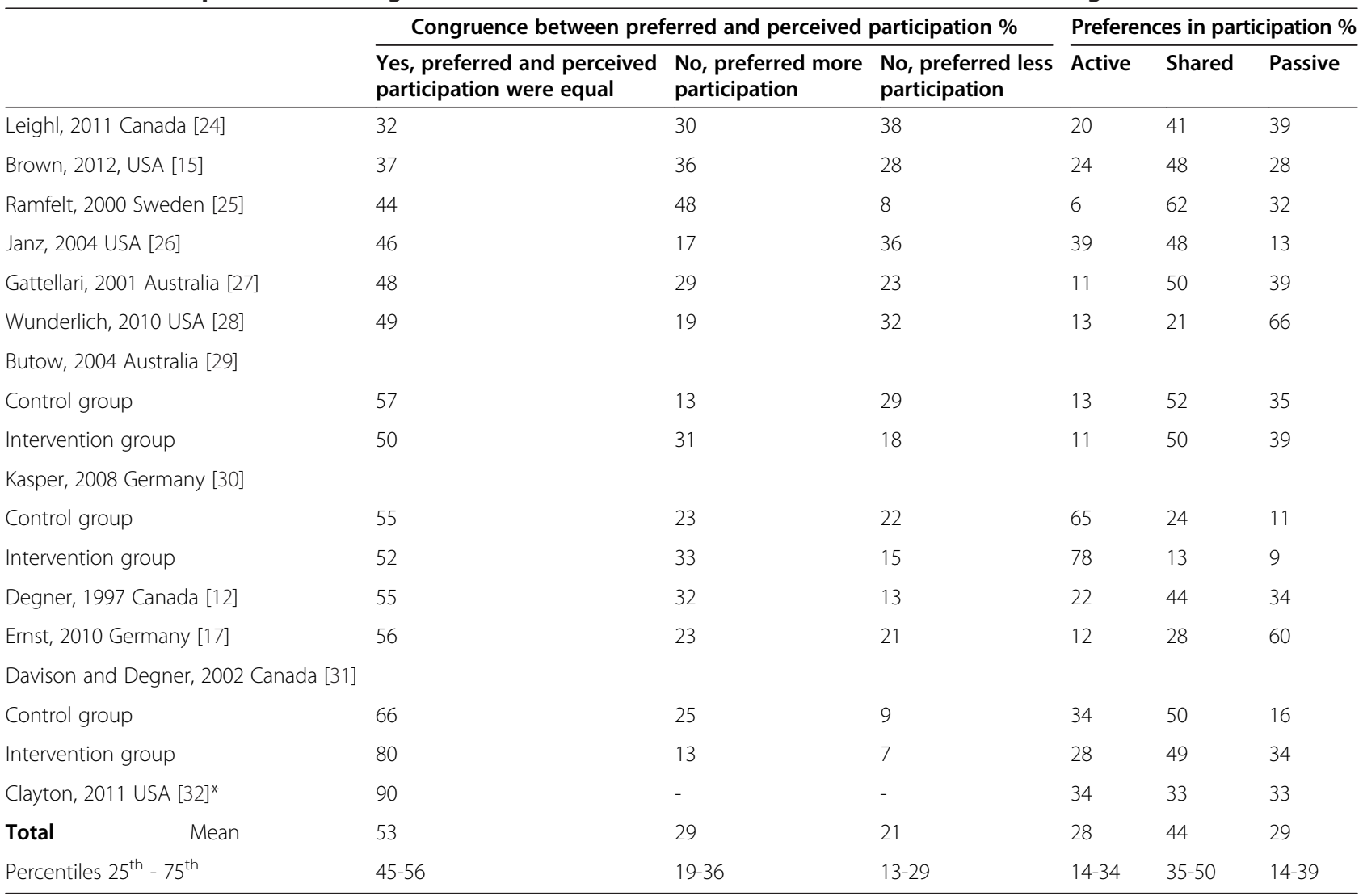

Preference prospectively measured ( $n=12$ studies, $n=15$ patient samples). Note *: studies reported\% of congruence, but not $\%$ for direction of mismatch. Table sorted on\% congruence.

Ten out of 12 studies included patients with cancer. Breast and colorectal cancer were the most common. In six studies preference for decision-making referred to a general preferences and in six studies preferences related to specific treatment options; operation (3 studies), medication ( 2 studies), screening (1 study).

Characteristics of the 32 retrospective studies (including 37 patient samples) are illustrated in Table 4. A total of 6088 women and 1709 men are represented in these studies (one study did not report gender, but included 2761 patients in total). Mean age was 56,4 years with an age range from 19 to 89 years old. Twenty-six out of 32 studies included patients with cancer. Breast cancer was the most common (14 studies). In 18 studies preference for treatment decision-making referred to general preference and in 14 studies preferences related specific treatment options; operation (9 studies), medication (3 studies), operation vs. medication (1 study), screening (1 study).

In total, 38 studies used the Control Preferences Scale to assess patient decision-making preferences and perceived participation; preferences were measured in various ways (questionnaire, face to face interview, telephone survey).
Most included studies were conducted in Europe (8 UK, 4 Germany, 4 Sweden, 4 Germany, 1 Belgium) followed by the United States of America (12 studies) and Canada (8 studies) and Australia (4 studies). Only 3 studies were conducted in Asia (2 studies in China, 1 in Japan) (Tables 3 and 4).

Five included studies were published before the year 2000, 11 between 2000 and 2005 and the majority (28 studies) after the year 2005. Furthermore, 31 out of 44 studies had a moderate score ( 2 or 3 out of 4 ) for the methodological quality assessed by the MMAT (23), 7 studies (including 3 RCTs) met all 4 criteria for methodological quality, and 6 studies had a low score (0 or 1$)$ for the methodological quality.

\section{Preferences}

Prospective vs. retrospective

In many studies, most patients preferred a shared role in the decision-making process (in 10 out of 15 patient samples of prospective studies and in 16 out of $37 \mathrm{pa}-$ tient samples of retrospective studies). In retrospective studies, the majority of patients preferred a passive role 
Table 2 Patients' preferences, congruence and direction of mismatch in medical decision-making

\begin{tabular}{|c|c|c|c|c|c|c|}
\hline & \multicolumn{3}{|c|}{ Congruence between preferred and perceived participation $\%$} & \multicolumn{3}{|c|}{ Preferences in participation $\%$} \\
\hline & $\begin{array}{l}\text { Yes preferred and perceived } \\
\text { participation were equal }\end{array}$ & $\begin{array}{l}\text { No, preferred more } \\
\text { participation }\end{array}$ & $\begin{array}{l}\text { No, preferred less } \\
\text { participation }\end{array}$ & Active & Shared & Passive \\
\hline Ramfelt et al., 2005 Sweden [33] & 31 & 55 & 14 & 18 & 47 & 35 \\
\hline Caress, 1997 UK [34] & 36 & 54 & 10 & 49 & 31 & 16 \\
\hline Sepucha et al., 2009 USA [22] & 38 & 46 & 16 & 8 & 88 & 4 \\
\hline Hack et al., 2006 Canada [35] & 48 & 41 & 12 & 36 & 42 & 23 \\
\hline Kremer et al., 2007 USA [36] & 49 & 25 & 25 & 28 & 59 & 13 \\
\hline Bilodeau et al., 1996 Canada [37]* & 50 & - & - & 20 & 37 & 43 \\
\hline Caress et al., 2005 UK & 51 & 43 & 6 & 24 & 36 & 40 \\
\hline \multicolumn{7}{|l|}{ Beaver and Booth, 1999 UK [38] } \\
\hline Colorectal cancer & 52 & 33 & 16 & 4 & 17 & 78 \\
\hline Breast cancer & 60 & 22 & 18 & 20 & 28 & 52 \\
\hline Purbrick et al., 2006 UK [39] & 56 & 18 & 26 & 10 & 69 & 21 \\
\hline Carey et al., 2012 Australia [40] & 56 & 34 & 10 & 26 & 30 & 45 \\
\hline Jefford et al., 2011 Australia [41] & 57 & 27 & 12 & 29 & 37 & 30 \\
\hline Nakashima et al., 2012 Japan [42] & 59 & 24 & 16 & 18 & 69 & 13 \\
\hline Ford et al., 2003 UK [43] & 60 & 25 & 15 & 18 & 46 & 36 \\
\hline Caldon, 2008 UK [44] & 61 & 9 & 29 & 40 & 42 & 17 \\
\hline \multicolumn{7}{|l|}{ Krist et al., 2007 USA [45]** } \\
\hline Control group & 61 & 23 & 16 & - & - & - \\
\hline Brochure group & 69 & 21 & 10 & - & - & - \\
\hline Website group & 73 & 16 & 11 & - & - & - \\
\hline Vogel et al., 2008 Germany [46] & 63 & 22 & 15 & 31 & 29 & 40 \\
\hline Wallberg et al., 2009 Sweden [47] & 66 & 27 & 7 & 10 & 68 & 23 \\
\hline \multicolumn{7}{|l|}{ Beaver et al., 2007 UK [48]* } \\
\hline Gyneacolocal cancer & 66 & 23 & 11 & 21 & 32 & 47 \\
\hline Breast cancer & 39 & - & - & 15 & 24 & 61 \\
\hline Colorectal cancer & 61 & - & - & 7 & 13 & 80 \\
\hline Hawley et al., 2007 USA [49] & 66 & 13 & 21 & - & - & - \\
\hline Zhang et al., 2011 China [50] & 69 & 28 & 3 & 11 & 46 & 44 \\
\hline Mahone, 2008 USA [19]* & 69 & - & - & 11 & 82 & 17 \\
\hline Lantz et al., 2005 USA [51]** & 69 & 11 & 20 & - & - & - \\
\hline Murray et al., 2007 UK [20] & 71 & 17 & 12 & 28 & 62 & 9 \\
\hline
\end{tabular}


Table 2 Patients' preferences, congruence and direction of mismatch in medical decision-making (Continued)

\begin{tabular}{|c|c|c|c|c|c|c|}
\hline Pardon et al., 2011 Belgium [52] & 71 & 20 & 9 & 15 & 22 & 63 \\
\hline Davidson et al., 1999 Canada [53] & 71 & 29 & 0 & 19 & 24 & 57 \\
\hline Wallberg et al., 2000 Sweden [54] & 72 & 20 & 8 & 13 & 21 & 66 \\
\hline Joliceur et al., 2009 Canada [55] & 77 & 23 & 0 & 15 & 54 & 31 \\
\hline Lam et al., 2003 China [18] & 80 & 7 & 13 & 33 & 59 & 8 \\
\hline Mohamedali et al., 2010 Canada [56]* & 80 & - & - & 11 & 52 & 37 \\
\hline Vogel et al., 2009 Germany [57] ${ }^{* *}$ & 84 & 2 & 15 & - & - & - \\
\hline Hawley et al., 2008 USA [58]** & 93 & 4 & 3 & - & - & - \\
\hline Palmer et al., 2012 USA [21] & 97 & 3 & 1 & 45 & 39 & 16 \\
\hline Mean & 63 & 24 & 13 & 21 & 43 & 36 \\
\hline Percentiles 25 - 75 & $54-71$ & $16-29$ & $8-16$ & $11-28$ & $29-59$ & $17-48$ \\
\hline
\end{tabular}

Preference retrospectively measured ( $\mathrm{n}=32$ studies, $\mathrm{n}=37$ patient samples).

Note *: Studies reported\% of congruence, but not\% for direction of mismatch.

Note **: Studies reported perceived participation.

Table sorted on\% congruence. 
Table 3 Study characteristics of 12 included prospective studies

\begin{tabular}{|c|c|c|c|c|c|c|}
\hline & \multicolumn{3}{|l|}{ Study population } & \multirow[t]{2}{*}{ Treatment decision } & \multirow{2}{*}{$\begin{array}{l}\text { Study design } \\
\text { Data collection }\end{array}$} & \multirow{2}{*}{$\begin{array}{l}\text { Quality of } \\
\text { studies** }\end{array}$} \\
\hline & Number (gender) & Age (mean) (range) & Patient population & & & \\
\hline \multicolumn{7}{|l|}{ Preference prospectively measured } \\
\hline \multirow[t]{2}{*}{ Brown et al., 2012 USA [15] } & 683 women & 55.3 & Breast cancer & General & RCT questionnaire before and after consultation & 3 \\
\hline & & - & & & & \\
\hline \multirow[t]{2}{*}{ Butow et al., 2004 Australia [29] } & 75 men & 58 & Cancer & General & RCT Questionnaires before and after consultation & 3 \\
\hline & 89 women & - & & & & \\
\hline \multirow[t]{2}{*}{ Clayton et al., 2011 USA [32] } & 62 men & 43.2 & \multirow{2}{*}{$\begin{array}{l}\text { Patients from general } \\
\text { practice }\end{array}$} & \multirow[t]{2}{*}{ General } & \multirow[t]{2}{*}{ self-reported data } & \multirow[t]{2}{*}{2} \\
\hline & 107 women & $18-89$ & & & & \\
\hline \multirow[t]{2}{*}{ Davison and Degner, 2002 Canada [31] } & 749 women & 58.3 & \multirow[t]{2}{*}{ Breast cancer } & \multirow[t]{2}{*}{ General } & \multirow{2}{*}{$\begin{array}{l}\text { Prospective, blocked, two-arm randomized } \\
\text { controlled trial }\end{array}$} & \multirow[t]{2}{*}{1} \\
\hline & & - & & & & \\
\hline \multirow[t]{2}{*}{ Degner et al., 1997 Canada [12] } & \multirow[t]{2}{*}{1012 women } & - & \multirow[t]{2}{*}{ Breast cancer } & \multirow[t]{2}{*}{ Operation } & \multirow{2}{*}{$\begin{array}{l}\text { Cross-sectional, consecutive sampling, nurse } \\
\text { administrated questionnaire }\end{array}$} & \multirow[t]{2}{*}{3} \\
\hline & & 58.3 & & & & \\
\hline \multirow[t]{2}{*}{ Ernst et al., 2010 Germany [17] } & 59 men & 57 & \multirow{2}{*}{$\begin{array}{l}\text { Hemato-oncological } \\
\text { illnesses }\end{array}$} & \multirow[t]{2}{*}{ General } & \multirow[t]{2}{*}{ Postal review interview } & \multirow[t]{2}{*}{1} \\
\hline & 45 women & $21-84$ & & & & \\
\hline \multirow[t]{2}{*}{ Gattellari et al., 2001 Australia [27] } & 133 men & 56.7 & \multirow[t]{2}{*}{ Cancer } & \multirow[t]{2}{*}{ General } & \multirow{2}{*}{$\begin{array}{l}\text { RCT prospective, cross-sectional consecutive } \\
\text { sampling self-administered questionnaire }\end{array}$} & 4 \\
\hline & 100 women & $22-82$ & & & & \\
\hline Janz et al., 2004 USA [26] & 101 women & 54.9 & Breast cancer & Operation & Telephone interviews & 4 \\
\hline & & $34-81$ & & & & \\
\hline Kasper et al., 2008 Germany [30] & 79 men & 43.1 & MS & Medication & RCT; telephone and post, standardized questionnaires & 4 \\
\hline & 218 women & - & & & & \\
\hline Leighl et al., 2011 Canada [24] & 62 men & 62.5 & Colorectal cancer & Medication & $\mathrm{RCT}$ & 2 \\
\hline & 38 women & - & & & & \\
\hline Ramfelt et al., 2000 Sweden [25] & 41 men & 70 & Colorectal cancer & Operation & Questionnaires & 3 \\
\hline & 45 women & $34-84$ & & & & \\
\hline Wunderlich et al., 2010 USA [28] & 134 men & 58 & Colorectal cancer & Screening & Pre- and post-visit survey & 1 \\
\hline & 229 women & - & & & & \\
\hline
\end{tabular}

Table sorted alphabetically.

Note **:Methodological quality of studies assessed with MMAT(23), ranging from 0 (lowest) to 4 (highest). 


\begin{tabular}{|c|c|c|c|c|c|c|}
\hline & \multicolumn{3}{|c|}{ Study population } & \multirow[t]{2}{*}{ Treatment decision } & \multirow{2}{*}{$\begin{array}{l}\text { Study design } \\
\text { Data collection }\end{array}$} & \multirow{2}{*}{$\begin{array}{l}\text { Quality of } \\
\text { studies** }\end{array}$} \\
\hline & $\begin{array}{l}\text { Number } \\
\text { (gender) }\end{array}$ & $\begin{array}{l}\text { Age (mean) } \\
\text { (range) }\end{array}$ & Patient population & & & \\
\hline \multicolumn{7}{|c|}{ Preference retrospectively measured } \\
\hline \multirow[t]{2}{*}{ Beaver et al., 1999 UK [48] } & 35 Men & 66.6 & \multirow[t]{2}{*}{ Colorectal cancer } & \multirow[t]{2}{*}{ General } & \multirow{2}{*}{$\begin{array}{l}\text { Cross-sectional, convenience } \\
\text { sampling structured interview schedule }\end{array}$} & \multirow[t]{2}{*}{2} \\
\hline & 13 Women & $23-83$ & & & & \\
\hline \multirow{2}{*}{$\begin{array}{l}\text { Beaver and Booth, } 2007 \\
\text { UK [38] }\end{array}$} & \multirow[t]{2}{*}{53 Women } & 55 & \multirow{2}{*}{$\begin{array}{l}\text { Gynaecological cancers breast } \\
\text { cancer colorectal cancer }\end{array}$} & \multirow[t]{2}{*}{ General } & \multirow[t]{2}{*}{ structured interviews, consecutive sample } & \multirow[t]{2}{*}{2} \\
\hline & & $24-82$ & & & & \\
\hline \multirow{2}{*}{$\begin{array}{l}\text { Bilodeau and Degner, } 1996 \\
\text { Canada [37] }\end{array}$} & \multirow[t]{2}{*}{74 Women } & - & \multirow[t]{2}{*}{ Breast cancer } & \multirow[t]{2}{*}{ Operation } & \multirow{2}{*}{$\begin{array}{l}\text { Cross-sectional, convenience sampling survey, } \\
\text { Interview schedule }\end{array}$} & \multirow[t]{2}{*}{2} \\
\hline & & $18-83$ & & & & \\
\hline \multirow[t]{2}{*}{ Caldon, 2008 UK [44] } & \multirow[t]{2}{*}{356 Women } & 58.5 & \multirow[t]{2}{*}{ Breast cancer } & \multirow[t]{2}{*}{ Operation } & \multirow{2}{*}{$\begin{array}{l}\text { Cross-sectional, convenience sampling } \\
\text { questionnaire survey }\end{array}$} & \multirow[t]{2}{*}{2} \\
\hline & & $30-89$ & & & & \\
\hline \multirow[t]{2}{*}{ Caress, 1997 UK [34] } & 245 Men & 47.2 & \multirow[t]{2}{*}{ Breast cancer } & \multirow[t]{2}{*}{ General } & Cross-sectional & 3 \\
\hline & 160 Women & $16-82$ & & & & \\
\hline Caress et al., 2005 UK [40] & 97 Men & 51.9 & Asthma & General & Cross sectional survey, structured interviews & 2 \\
\hline & 133 Women & $19-94$ & & & & \\
\hline Carey et al., 2012 Australia [16] & 158 Men & 59.5 & Haemato-logical cancer & General & Cross-sectional design, questionnaire & 2 \\
\hline & 110 Women & - & & & & \\
\hline Davidson et al., 1999 & 10 Men & 65 & Lung cancer & General & Interview & 2 \\
\hline Canada [53] & 11 Women & - & & & & \\
\hline Ford et al., 2003 UK [43] & 56 Men & 49 & Patients from general & General & Questionnaire & 3 \\
\hline & 115 Women & $16-88$ & practıce & & & \\
\hline Hack et al., 2006 & 205 Women & 59.5 & Breast cancer & Operation & Interviews & 2 \\
\hline Canada [35] & & - & & & & \\
\hline Hawley et al., 2007 & 1101 Women & 59 & Breast cancer & Operation & A self-administered survey of a & 3 \\
\hline [49] & & $29-79$ & & & po & \\
\hline Hawley et al., 2008 & 877 Women & 59 & Breast cancer & Operation & Survey, data questionnaire & 3 \\
\hline USA [58]* & & $29-79$ & & & & \\
\hline Jefford et al., 2011 & 68 Men & 58.4 & Cancer & General & Convenience sample, questionnaires & 3 \\
\hline Australıa [41] & 34 Women & $29-85$ & & & & \\
\hline
\end{tabular}


Table 4 Study characteristics 32 included retrospective studies (Continued)

\begin{tabular}{|c|c|c|c|c|c|c|}
\hline $\begin{array}{l}\text { Joliceur et al., } 2009 \\
\text { Canada [55] }\end{array}$ & 13 Women & $\begin{array}{l}57 \\
46-77\end{array}$ & Ovarian cancer & General & $\begin{array}{l}\text { Retrospective cross-sectional design. Face } \\
\text { to face interviews (semi-structured) }\end{array}$ & 2 \\
\hline \multirow{2}{*}{$\begin{array}{l}\text { Kremer et al., } 2007 \\
\text { USA [36] }\end{array}$} & 51 Men & 42 & \multirow[t]{2}{*}{ HIV } & \multirow[t]{2}{*}{ Medication } & \multirow[t]{2}{*}{ Cross-sectional study } & \multirow[t]{2}{*}{2} \\
\hline & 28 Women & - & & & & \\
\hline \multirow[t]{2}{*}{ Krist et al., 2007 USA [45] } & \multirow[t]{2}{*}{497 Men } & 57 & \multirow[t]{2}{*}{ Prostate cancer } & \multirow[t]{2}{*}{ Screening } & \multirow[t]{2}{*}{ RCT } & \multirow[t]{2}{*}{4} \\
\hline & & $50-70$ & & & & \\
\hline \multirow[t]{2}{*}{ Lam et al., 2003 China [18] } & \multirow[t]{2}{*}{154 Women } & 59 & \multirow[t]{2}{*}{ Breast cancer } & \multirow[t]{2}{*}{ Operation } & \multirow[t]{2}{*}{ Face-to-face interviews } & \multirow[t]{2}{*}{2} \\
\hline & & $28-79$ & & & & \\
\hline \multirow[t]{2}{*}{ Lantz et al., 2005 USA* [51] } & 1633 Women & - & \multirow[t]{2}{*}{ Breast cancer } & \multirow[t]{2}{*}{ Operation } & \multirow[t]{2}{*}{ Cross-sectional, mailed survey } & \multirow[t]{2}{*}{3} \\
\hline & & - & & & & \\
\hline \multirow[t]{2}{*}{ Mahone, 2008 USA [19] } & 49 Men & 43 & \multirow[t]{2}{*}{ Serious mental illness } & \multirow[t]{2}{*}{ Medication } & Cross-sectional, correlational study & 1 \\
\hline & 35 Women & $20-62$ & & & & \\
\hline Mohamedali et al., 2010 & 18 Men & - & AML & General & Questionnaires & 4 \\
\hline Canada [56] & 17 Women & & & & & \\
\hline Murray et al., 2007 UK [20] & 2761 & - & American Public & General & Cross-sectional telephone survey & 2 \\
\hline & & - & & & & \\
\hline Nakashima et al., 2012 & 104 Women & - & Breast cancer & General & Cross-sectional design, questionnaires & 2 \\
\hline & & - & & & & \\
\hline Palmer et al., 2012 & 181 Men & 61.3 & Prostate cancer & General & Cross-sectional case-control study, interview & 3 \\
\hline & & $43-75$ & & & & \\
\hline Pardon et al., 2011 & 102 Men & - & Lung cancer & General & Questionnaire & 4 \\
\hline & 26 Women & 64.4 & & & & \\
\hline Purbrick et al., 2006, & 12 Men & 63 & Ocular cancer & Operation & Questionnaire & 0 \\
\hline UK [39] & 27 Women & $19-80$ & & & & \\
\hline Ramfelt et al., 2005 & 26 Men & 69 & Rectal cancer & Operation & Prospective, cross-sectional convenience sampling & 2 \\
\hline Sweden [33] & 29 Women & $34-83$ & & & & \\
\hline Sepucha et al., 2009 & 32 Women & 55 & Breast cancer & General & Patient survey & 3 \\
\hline USA [2L] & & $37-78$ & & & & \\
\hline Vogel et al., 2008 & 137 Women & 53.75 & Breast cancer & General & Self-explanatory questionnaire & 3 \\
\hline Germany [46] & & $19-75$ & & & & \\
\hline
\end{tabular}


Table 4 Study characteristics 32 included retrospective studies (Continued)

\begin{tabular}{|c|c|c|c|c|c|c|}
\hline \multirow{2}{*}{$\begin{array}{l}\text { Vogel et al., } 2009 \\
\text { Germany* [57] }\end{array}$} & \multirow[t]{2}{*}{135 Women } & 54 & \multirow[t]{2}{*}{ Breast cancer } & \multirow{2}{*}{$\begin{array}{l}\text { Mastectomy vs breast-concerving } \\
\text { therapy vs chemotherapy }\end{array}$} & \multirow{2}{*}{$\begin{array}{l}\text { Consecutive sample self-administered } \\
\text { questionnaire }\end{array}$} & \multirow[t]{2}{*}{3} \\
\hline & & $19-75$ & & & & \\
\hline \multirow{2}{*}{$\begin{array}{l}\text { Wallberg et al., } 2000 \\
\text { Sweden [54] }\end{array}$} & 201 Women & - & \multirow[t]{2}{*}{ Breast cancer } & \multirow[t]{2}{*}{ General } & \multirow[t]{2}{*}{ Interviews } & \multirow[t]{2}{*}{4} \\
\hline & & - & & & & \\
\hline \multirow{2}{*}{$\begin{array}{l}\text { Wallberg et al., } 2009 \\
\text { Sweden [47] }\end{array}$} & 201 Women & 60.7 & \multirow[t]{2}{*}{ Breast cancer } & \multirow[t]{2}{*}{ Medication } & \multirow[t]{2}{*}{ Questionnaires } & \multirow[t]{2}{*}{1} \\
\hline & & $55.3-$ & & & & \\
\hline \multirow{2}{*}{$\begin{array}{l}\text { Zhang et al., } 2011 \\
\text { China [50] }\end{array}$} & 104 Men & 45 & \multirow[t]{2}{*}{ Chronic hepatitis } & \multirow[t]{2}{*}{ General } & \multirow[t]{2}{*}{ Cross-sectional, mailed survey } & \multirow[t]{2}{*}{3} \\
\hline & 74 Women & $18-69$ & & & & \\
\hline
\end{tabular}

Note *:Studies reported perceived participation.

Note *:Methodological quality of studies assessed with MMAT (23), ranging from 0 (lowest) to 4 (highest). Table sorted alphabetically. 
Table 5 Reported significant associations with preferred role, perceived role and congruence

\begin{tabular}{|c|c|c|}
\hline Determinant & Associations studied for & Outcome \\
\hline \multirow[t]{8}{*}{ Age $(N=16)$} & Preferred role $(n=13)$ & - Older prefer more passive role $[20,35,40,53,54]$ \\
\hline & & - Younger prefer a more active role $[12,35,44,50]$ \\
\hline & & - Younger prefer more often shared role $[18,35,54]$ \\
\hline & & - No association found $[33,37,38]$ \\
\hline & Perceived role $(n=3)$ & - Older women perceived a more passive role [37] \\
\hline & & - Younger perceived less active [49] \\
\hline & & - No association found [44] \\
\hline & Congruence $(n=1)$ & - No association found [17] \\
\hline Gender $(N=1)$ & Congruence $(n=1)$ & - No association found [17] \\
\hline \multirow[t]{5}{*}{ Education level $(\mathrm{N}=10)$} & Preferred role $(n=7)$ & - Higher educated prefer more often active role $[12,20,26,42,54]$ \\
\hline & & - Lower educated patients prefer more often passive role $[40,50]$ \\
\hline & Perceived role $(n=1)$ & - No association found [35] \\
\hline & Congruence $(n=2)$ & - If high school or less; patients preferred less involvement [49] \\
\hline & & - No association found [17] \\
\hline Socioeconomic status $(\mathrm{N}=1)$ & Preferred role $(n=1)$ & - Higher income prefer more active [22] \\
\hline \multirow[t]{2}{*}{ Ethnicity $(N=2)$} & Preferred role $(n=1)$ & - Black patients (vs. white) prefer more passive [20] \\
\hline & Perceived role $(n=1)$ & - Latina-Spanish speaking women preferred more involvement [58] \\
\hline \multirow[t]{3}{*}{ Marital status $(\mathrm{N}=3)$} & Preferred role $(n=3)$ & - If partner than more often preference for a shared or passive role [36] \\
\hline & & - Widowed more like to prefer passive role [35] \\
\hline & & $\begin{array}{l}\text { - Married, (who had lumpectomy and whose first language was English) } \\
\text { prefer more active/shared roles [12] }\end{array}$ \\
\hline QoL $(N=2)$ & Congruence $(n=2)$ & - No association found $[52,57]$ \\
\hline \multirow[t]{3}{*}{ Depression/Anxiety $(\mathrm{N}=3)$} & Preference $(n=1)$ & - Patients who preferred a passive role were more depressed [46] \\
\hline & Congruence $(n=2)$ & - Lower depression scores if congruence [57] \\
\hline & & - Mismatch not associated with changes in anxiety levels [27] \\
\hline
\end{tabular}

more often than in the prospective studies (in 12 out of 37 retrospective patient samples and 2 out of 15 prospective patient samples) (Tables 1 and 2).

\section{Cancer vs. non-cancer}

Overall, patients with cancer more often desired a passive role than patients without cancer (14 out of 43 patient samples with cancer vs. zero out of six patient samples with non-cancer).

\section{General vs. specific treatment options}

When preferences were asked in general patients often desired a passive role (12 out of 29 patient samples). If preferences related to a specific treatment option were assessed (e.g., for example breast cancer surgery) patients less often desired a passive role ( 2 out of 22 patient samples).

\section{Congruence}

All studies showed discrepancies between preferred and perceived roles in medical decision-making, shown in Table 1 and Table 2. The overall mean of congruence (all studies) was $60 \%$ (49 and 70 representing the $25^{\text {th }}$ and $75^{\text {th }}$ percentiles, respectively).

\section{Prospective vs. retrospective}

The percentages of congruence was slightly higher in retrospective studies than in prospective studies (mean $63 \%$ vs. mean $53 \%$ ).

Where no congruence was found, in 36 patient samples most patients preferred more participation than perceived. In the prospective studies, 11 out of 15 patient samples preferred more participation than perceived and in the retrospective studies, 25 out of 33 .

In 9 patient samples most patients preferred less participation than perceived. In the prospective studies, 3 out of 15 patient samples preferred less participation and in the retrospective studies, 6 out of 37 . The latter all involved patients with cancer $[18,26,28,29,39,44,49,51,57]$.

\section{Cancer vs. non-cancer}

Congruence was similar for patients with cancer and for patients with non-cancer (60\% and 59\%, respectively). 


\section{General vs. specific treatment options}

There was no difference in congruence between treatment preference asked in general and for specific treatment options (58\% and $61 \%$, respectively).

\section{Associations}

Table 5 summarizes the factors associated with patient participation in medical decision-making, distinguishing associations with preferred role, perceived role and congruence.

\section{Age}

Associations between age and preferred role were reported in 13 different studies. Six studies on various patient populations (women with breast cancer [35,54], patients with lung cancer [53], patients with asthma [40] patients visiting their GP [43] and general public [20] found that older patients preferred more often a passive role in medical decision-making, four studies (three among patients with breast cancer $[12,35,44]$ and one among patients with chronic hepatitis [50] found that younger patients preferred more often an active role, and three studies (all among patients with breast cancer $[18,35,54]$ found that younger patients preferred more often a shared role. Finally, three studies (one among patients with breast cancer [37], one among patients with colorectal cancer [33] and one among patients with different types of cancer [38] found no association between age and preferred role in medical decision-making.

Three studies reported associations between age and perceived role (all among patients with breast cancer) of which one found that older women perceived a more passive role than preferred [37], one found that younger patients perceived a less active role than preferred [49] and one found no association [44].

One study reported no association between age and congruence was found [17].

\section{Gender}

One study in patients with haemato-oncological illnesses [17] reported no association between gender and congruence between preferred and perceived participation.

\section{Education level}

Seven studies reported on associations between education and preferred role using different definitions of higher education (for instance 'high school completed or higher' [12], 'bachelor degree' [26], 'college degree' [42]).

Five studies (four among patients with breast cancer $[12,26,42,54]$ and one among the general public [20]) found that higher educated people more often prefer an active role, two studies (one among patients with breast cancer [50] and one among patients with asthma [40]) found that lower educated people more often prefer a passive role. One study among patients with breast cancer reported no association between education and perceived role [35].

Two studies reported on association between education and congruence of which one among patients with breast cancer [49] found that lower educated people preferred less participation than perceived and the other study among patient with solid and haematological cancer [17] did not find an association between education and congruence.

\section{Socioeconomic status}

One study among the general public [20] reported that patients with a higher income more often preferred an active role.

\section{Ethnicity}

One study reported that black patients prefer a more passive role compared to white patients [20] and one study reported that Latina-Spanish speaking women with breast cancer [58] preferred more involvement than perceived in the decision-making process compared to Latina-English speaking, African American and Caucasian women with breast cancer.

\section{Marital status}

An association between marital status and preferred role repoted in three studies. Of these, one study among patients with breast cancer [35] reported that widowed women preferred more often a passive role in medical decision-making than partnered, divorced, separated or never married women, another study among women with breast cancer [12] found that women who were married were more likely to prefer active or collaborative roles in treatment decisions, and one study among patient with HIV/AIDS [36] showed that patients with a partner preferred a less active role than patients not having a partner.

\section{Quality of life}

Two studies reported no association between quality of life and congruence. One among patients with primary breast cancer [57] and one among patients with advanced lung cancer [52].

\section{Depression or anxiety}

One study [46] showed that patients with primary breast cancer with a preference for a passive role in decision making were more depressed than patients with a preference for active role in decision making. A sample of patients with breast cancer [57] showed that patients who participated in decision making as much as they preferred had lower depression scores than patients who participated less than preferred. 
A study among patients with different types of cancer [27] showed that role mismatch was associated with changes in anxiety before and directly after consultation with their oncologist.

\section{Interventions}

Four randomized controlled trials reported results concerning preferred and perceived role in medical decision making.

One study included patients with multiple sclerosis evaluating an evidence-based patient decision aid on multiple sclerosis immunotherapy [30]. The percentage of congruence between preferred and perceived role during consultation did not differ between the group with decision aid and group with standard information.

A study investigated patient education on prostate cancer screening among men who underwent a health maintenance examination at a family practice, and its influence on involvement in decision making [45]. One group received a web based decision aid, another group received a paper based decision aid and a third (control) group received no previsit education. Patients with either decision aid more often perceived an active role in decision making compared to the control group.

One study evaluated the feasibility of using a computer intervention to enhance communication between health care professionals and women with breast cancer [31]. Women in the intervention group were encouraged to use the information and decision preferences profiles generated by the computer program at their clinic appointment and women in the control group simply completed measures of decision preference before their clinic appointment. A significantly higher number of women in the intervention group reported that their perceived role was more passive than they preferred beforehand.

One study evaluated a cancer consultation preparation package (CCPP) designed to facilitate patient involvement in oncology consultations in men and women with different types of cancer [29]. Patients received either this CCPP with a question prompt sheet, booklet on clinical decisions and patient rights and introduction to the clinic, or a control booklet and introduction to the clinic. Patients that received the CCPP were less likely to receive their preferred role in decision making than the control group.

\section{Discussion and conclusion Discussion}

This study reviews the findings of 44 studies (including 52 patient samples) reporting on patients' preferences for participation in medical decision-making and congruence with perceived participation. Commonly, most patients preferred a shared role in the decision-making process. However, in studies that assessed preferences retrospectively, the majority of patients more often preferred a passive role. Also, patients with cancer more often desired a passive role than non-cancer patients and when preferences were asked in general, patients more often desired a passive role than when asked about more specific treatment option.

We found that the mean of patients whose perceived role in decision-making was congruent with their desired role was $60 \%$. In case of no congruence, patients in general preferred more participation in the decisionmaking process than perceived. Furthermore, this review showed that in studies in which preference was retrospectively measured congruence was more often found, compared to the prospective studies. Percentages of congruence was similar for patients with cancer and patients without cancer and also regardless of whether preferences related to general or specific treatment options. Lastly, several associations were reported with patient preference for participation in medical decision-making, most commonly age and education level, and most frequently reporting that older people and people with a lower education prefer more often a passive role.

This review gives health care professionals (more) insight in patient wishes and experiences with treatment decision-making and could help them guide patients in treatment decision-making.

\section{Strengths and limitations}

A strength of this review is the broad approach. Most research concentrates on patient participation in cancer decisions, but treatment decision-making is relevant to a much broader range of patients than cancer care alone. Therefore we included all patient populations (e.g. patients with cancer patients and patients with other conditions). Furthermore, in addition to previous reviews, we drew distinctions between prospective and retrospective measurement of preferences and type of treatment decisions (i.e., general or specific).

Although this review covers a substantial number of studies on patient preference for participation in decisionmaking, it also has limitations. First, studies included in this review were only those in the English language, and although covering most parts of the world, they are conducted in wealthy countries. This limits the generalizability of our findings. Secondly, we only included studies in which congruence was given or could be calculated. Therefore, we did not retrieve all studies relevant to preferences. Third, we recoded the reported data on patient participation on a 5-point scale into three categories (active, shared or passive) before calculating congruence, this may have caused an overestimation of congruence.

Preferences and congruence with perceived participation Overall our findings are in accordance with the findings of Tariman and colleagues among patients with cancer 
[10]. While our study included over 10 studies published subsequent to their review and 8 studies among patients with other conditions: in most studies a majority of people prefer a shared role, in all studies there is a group in which there is a mismatch between preferred and perceived role and in case of this mismatch this is more often in the direction of preferring more participation than perceived. Despite of this overall accordance in findings, our review shows that there seems to be some differences between patients with cancer and patients with other diagnoses. Patients with cancer appeared to more frequently prefer a passive role in participations, while there appeared to be no differences in the occurrence of mismatch. Yet when a mismatch occurred, it concerned patients with cancer who preferred less participation than those with other conditions $[18,26,28,29,39,44,49,51,57]$. It should be noted that decisions in cancer care may differ from non-cancer diseases. Ernst et al. [17] argue that preferences for a passive role in haematology patients may be a consequence of stress as a result of the invasive and complex therapies.

While in case of a mismatch generally patients preferred a more active role (in 36 patient samples), it is important not to forget that we found nine patient samples in which more patients with a mismatch preferred a less active role than perceived. Further, in all studies there were patients that preferred less participation. For these patients, the idea that generally more participation should be advocated can be questioned. Age and educational level were most frequently associated with preferred level of participation in medical decision-making, finding that older patients and lower educated patients preferred a less active role. Simply following these preferences, might cause inequity in health care provision, as older and lower educated patients are often the most vulnerable people. In order to foster the health of these patients, it might be necessary to promote their participation and empower them to take an active role in decision-making. Studies showed that active participation was associated with positive health outcomes such as overall quality of life, higher physical and social functioning and less fatigue [35]. Yet, one should also be aware of possible negative effects. Some studies showed that pushing patients to play an active role in medical decisions could have negative consequences such as decisional regret [51], increased anxiety [59] or less confidence that they make the right treatment decision and unnecessary stress [18].

\section{Prospective versus retrospective assessment of preference}

We found some differences between the 12 studies in which preferences were measured before the decisionmaking took place (prospectively) and the 37 studies in which preferences were measured after the decision- making took place (retrospectively). This suggests that there is indeed a fundamental difference between the two ways of studying preferences. One difference we found was that when preference was assessed retrospectively, patients seemed more frequently prefer a passive role in decision-making. This is in line with a study that found that relying on the physicians' expertise and trust in the physician can play a role in preferences regarding patient participation [60].

Another difference we found is that mismatch less often was found in retrospective studies than in prospective studies. This is not surprising given that in retrospective studies the assessment of preference probably includes an element of evaluation of the decision-making that occurred. This may be due to patients' desire for congruence, introducing cognitive bias into the response. Yet, this potential desire for congruence did not result in $100 \%$ congruence. That mismatch is found in retrospective assessment of preferences in part of the patients suggests that patients are able to separate preference from evaluation or satisfaction with the decision-making at least to some extent. Taking this into account one could argue that retrospective assessment of preference might be as suitable as prospective assessment of preferences. Practically it is easier to measure preferred and perceived participation at the same moment. More substantially, compared to prospective assessment it takes the evolution of preferences into account, since preferences can evolve during the decision-making process, as reported by several authors $[15,61]$. On the other hand, prospective measurement of course holds the benefit of most accurate assessing preferences.

\section{Conclusion}

This review shows that patients' preferences for participation in medical decision-making vary. This review also shows that congruence is achieved in about $60 \%$ of the cases, and that in cases in of mismatch, patients most often preferred more involvement. Nevertheless, there was also a substantial group of patients that preferred less involvement than perceived. These results hold both for studies that measured preferences prospectively and retrospectively. Yet we did find that there are differences between the two methods of measuring preferences, it appears that the experience of the decision-making influence retrospective measurement. This leads to somewhat higher proportions of people preferring a passive role and more frequent mismatch in retrospective measurement compared to prospective measurement. Although some studies found associations with socio-demographics, patient preference for participation in medical decisionmaking seems individual. Therefore it is important that physicians discuss the preferred role with their patient in order to fit the decision-making process to the needs and capabilities of the individual patient. 


\section{Competing interests}

The authors declare that there is no conflict of interest.

\section{Authors' contributions}

All authors ( $L B, W H, D R M T, G A M W, H R W P$ and BDOP) participated in the conception and design of this review. $L B$ and $W H$ reviewed the titles and abstracts. $L B, W H, H R W P$ and BDOP reviewed the full papers to determine which to include in the review. All authors were involved in the interpretation of the findings. All authors were involved in either drafting the manuscript or providing revisions. All authors read and approved the final manuscript.

\section{Acknowledgements}

The authors would like to thank R.H.J. Otten of the Medical Library, VU university, for his assistance with developing a the search strategy and searching the computerized bibliographic databases.

\section{Role of funding}

This study was funded by the Innovational Research Incentives Scheme VICl 2008 from the Netherlands Organisation for Scientific Research (NWO).

\section{Author details}

${ }^{1}$ Department of Public and Occupational Health, EMGO Institute for Health and care research, VU University Medical Center, Van der Boechorststraat 7, 1081 Amsterdam, BT, The Netherlands. ²Department of Medical Humanities, EMGO Institute for Health and care research, VU University Medical Center, Amsterdam, The Netherlands.

Received: 5 September 2013 Accepted: 27 March 2014

Published: 3 April 2014

\section{References}

1. Harrington J, Noble LM, Newman SP: Improving patients' communication with doctors: a systematic review of intervention studies. Patient Educ Couns 2004, 52(1):7-16.

2. Kinnersley P, Edwards A, Hood K, Ryan R, Prout H, Cadbury N, MacBeth F, Butow $P$, Butler $C$ : Interventions before consultations to help patients address their information needs by encouraging question asking: systematic review. BMJ 2008, 337:a485.

3. Cegala DJ, Post DM: The impact of patients' participation on physicians' patient-centered communication. Patient Educ Couns 2009, 77(2):202-208.

4. Cribb A, Entwistle VA: Shared decision making: trade-offs between narrower and broader conceptions. Health Expect 2011, 14(2):210-219.

5. Kiesler DJ, Auerbach SM: Optimal matches of patient preferences for information, decision-making and interpersonal behavior: evidence, models and interventions. Patient Educ Couns 2006, 61(3):319-341.

6. Hamann J, Mendel R, Buhner M, Kissling W, Cohen R, Knipfer E, Eckstein HH: How should patients behave to facilitate shared decision making - the doctors' view. Health Expect 2011, 30:10-7625.

7. Wigersma L, Brabers A, Reitsma M, Jong JD: Transparantie is niet zichtbaar: onderzoek naar opvattingen over vraagsturing in de zorg. Medisch Contact 2011, 66(45):2776-2778.

8. Degner LF, Sloan JA, Venkatesh P: The control preferences scale. Can J Nurs Res 1997, 29(3):21-43.

9. Chewning B, Bylund CL, Shah B, Arora NK, Gueguen JA, Makoul G: Patient preferences for shared decisions: a systematic review. Patient Educ Couns 2012, 86(1):9-18.

10. Tariman JD, Berry DL, Cochrane B, Doorenbos A, Schepp K: Preferred and actual participation roles during health care decision making in persons with cancer: a systematic review. Ann Oncol 2010, 21(6):1145-1151.

11. Arora NK, McHorney CA: Patient preferences for medical decision making: who really wants to participate? Med Care 2000, 38(3):335-341.

12. Degner LF, Kristjanson LJ, Bowman D, Sloan JA, Carriere KC, O'Neil J, Bilodeau B, Watson P, Mueller B: Information needs and decisional preferences in women with breast cancer. JAMA 1997, 277(18):1485-1492.

13. Deber RB, Kraetschmer N, Urowitz S, Sharpe N: Do people want to be autonomous patients? Preferred roles in treatment decision-making in several patient populations. Health Expect 2007, 10(3):248-258.

14. Cohen $\mathrm{H}$, Britten $\mathrm{N}$ : Who decides about prostate cancer treatment? A qualitative study. Fam Pract 2003, 20(6):724-729.

15. Brown R, Butow P, Wilson-Genderson M, Bernhard J, Ribi K, Juraskova I: Meeting the decision-making preferences of patients with breast cancer in oncology consultations: impact on decision-related outcomes. J Clin Oncol 2012, 30(8):857-862.

16. Carey M, Anderson A, Sanson-Fisher R, Lynagh M, Paul C, Tzelepis F: How well are we meeting haematological cancer survivors' preferences for involvement in treatment decision making? Patient Educ Couns 2012, 88(1):87-92.

17. Ernst J, Kuhnt S, Schwarzer A, Aldaoud A, Niederwieser D, Mantovani-Loffler $L$, Kuchenbecker D, Schroder C: The desire for shared decision making among patients with solid and hematological cancer. Psychooncology 2011, 20(2):186-193.

18. Lam W, Fielding R, Chan M, Chow L, Ho E: Participation and satisfaction with surgical treatment decision-making in breast cancer among Chinese women. Breast Cancer Res Treat 2003, 80(2):171-180.

19. Mahone $\mid \mathrm{H}$ : Shared decision making and serious mental illness. Arch Psychiatr Nurs 2008, 20(2):16-93.

20. Murray E, Pollack L, White M, Lo B: Clinical decision-making: Patients' preferences and experiences. Patient Educ Couns 2007, 65(2):189-196.

21. Palmer N, Tooze J, Turner A, Xu J, Avis N: African American prostate cancer survivors' treatment decision-making and quality of life. Patient Educ Couns 2012, 90(1):61-68

22. Sepucha KR, Ozanne EM, Partridge AH, Moy B: Is there a role for decision aids in advanced breast cancer? Med Decis Making 2009, 29(4):475-482.

23. Pluye $P$, Robert $E$, Cargo M, Barlett G, OCathain A, Griffiths F, Boardman F, Gagnon MP, Rousseau MC: Proposal: a mixed methods appraisal tool for systematic mixed studies reviews. 2011. Retrieved on 14-1-2014 from: http://mixedmethodsappraisaltoolpublic.pbworks.com.

24. Leighl NB, Shepherd HL, Butow PN, Clarke SJ, McJannett M, Beale PJ, Wilcken NR, Moore MJ, Chen EX, Goldstein D, Horvath L, Knox JJ, Krzyzanowska M, Oza AM, Feld R, Hedley D, Xu W, Tattersall MH: Supporting treatment decision making in advanced cancer: a randomized trial of a decision aid for patients with advanced colorectal cancer considering chemotherapy. J Clin Oncol Official J Am Soc Clin Oncol 2011, 29(15):2077-2084.

25. Ramfelt E, Langius A, Bjorvell H, Nordstrom G: Treatment decision-making and its relation to the sense of coherence and the meaning of the disease in a group of patients with colorectal cancer. Eur J Cancer Care (Engl) 2000, 9(3):158-165.

26. Janz NK, Wren PA, Copeland LA, Lowery JC, Goldfarb SL, Wilkins EG Patient-physician concordance: preferences, perceptions, and factors influencing the breast cancer surgical decision. J Clin Oncol 2004, 22(15):3091-3098.

27. Gattellari M, Butow PN, Tattersall MHN: Sharing decisions in cancer care. Soc Sci Med 2001, 52(12):1865-1878.

28. Wunderlich T, Cooper G, Divine G, Flocke S, Oja-Tebbe N, Stange K, Lafata JE: Inconsistencies in patient perceptions and observer ratings of shared decision making: the case of colorectal cancer screening. Patient Educ Couns 2010, 80(3):358-363.

29. Butow P, Devine R, Boyer M, Pendlebury S, Jackson M, Tattersall MH: Cancer consultation preparation package: changing patients but not physicians is not enough. J Clin Oncol Official J American Soc Clin Oncol 2004, 22(21):4401-4409.

30. Kasper J, Köpke S, Mülhauser I, Nübling M, Heesen C: Informed shared decision making about immunotherapy for patients with multiple sclerosis (ISDIMS): a randomized controlled trial. Euro J Neurol 2008, 15(12):1345-1352.

31. Davison BJ, Degner LF: Feasibility of using computer-assisted intervention to enhance the way women with breast cancer communicate with their physicians. Cancer Nurs 2002, 25(6):417-424

32. Clayton MF, Latimer S, Dunn TW, Haas L: Assessing patient-centered communication in a family practice setting: how do we measure it, and whose opinion matters? Patient Educ Couns 2011, 84(3):294-302.

33. Ramfelt E, Lutzen $\mathrm{K}$, Nordstrom G: Treatment decision-making in a group of patients with colo-rectal cancer before surgery and a one-year follow-up. Eur J Cancer Care (Engl) 2005, 14(4):327-335.

34. Caress AL: Patient roles in decision-making. Nurs Times 1997, 93(31):45-48.

35. Hack TF, Degner LF, Watson P, Sinha L: Do patients benefit from participating in medical decision making? Longitudinal follow-up of women with breast cancer. Psychooncology 2006, 15(1):9-19.

36. Kremer H, Ironson G, Schneiderman N, Hautzinger M: "It's my body": does patient involvement in decision making reduce decisional conflict? Med Decis Making 2007, 27(5):522-532. 
37. Bilodeau BA, Degner LF: Information needs, sources of information, and decisional roles in women with breast cancer. Oncol Nurs Forum 1996, 23(4):691-696

38. Beaver K, Booth K: Information needs and decision-making preferences: comparing findings for gynaecological, breast and colorectal cancer. Eur J Oncol Nurs 2007, 11(5):409-416.

39. Purbrick RMJ, Tu KL, Damato BE: The patient's role in the decision-making process-a perspective from the liverpool ocular oncology centre. Eye (Lond) 2006, 20(9):1034-1039.

40. Caress AL, Beaver K, Luker K, Campbell M, Woodcock A: Involvement in treatment decisions: what do adults with asthma want and what do they get? Results of a cross sectional survey. Thorax 2005, 60(3):199-205.

41. Jefford M, Mileshkin L, Matthews J, Raunow H, O'Kane C, Cavicchiolo T, Brasier H, Anderson M, Reynolds J: Satisfaction with the decision to participate in cancer clinical trials is high, but understanding is a problem. Support Care Cancer 2010, 19(3):371-379.

42. Nakashima M, Kuroki S, Shinkoda H, Suetsugu Y, Shimada K, Kaku T: Information-seeking experiences and decision-making roles of Japanese women with breast cancer. Fukuoka lgaku Zasshi 2012, 103(6):120-130.

43. Ford S, Schofield T, Hope T: Are patients' decision-making preferences being met? Health Expect 2003, 6(1):72-80

44. Caldon LMM, Walters SJ, Reed MWR: Changing trends in the decisionmaking preferences of women with early breast cancer. Br J Surg 2008, 95(3):312-318.

45. Krist AH, Woolf SH, Johnson RE, Kerns JW: Patient education on prostate cancer screening and involvement in decision making. Ann Fam Med 2007, 5(2):112-119.

46. Vogel BA, Helmes AW, Hasenburg A: Concordance between patients' desired and actual decision-making roles in breast cancer care. Psychooncology 2008, 17(2):182-189.

47. Wallberg B, von SE Bolund C, Bergh J, Wilking N: Hormone replacement therapy after breast cancer: attitudes of women eligible in a randomized trial. Climacteric J Int Menopause Soc 2009, 12(6):478-489.

48. Beaver K, Bogg J, Luker K: Decision-making role preferences and information needs: a comparison of colorectal and breast cancer. Health Expect 1999, 2(4):266-276.

49. Hawley ST, Lantz PM, Janz NK, Salem B, Morrow M, Schwartz K, Liu L, Katz SJ: Factors associated with patient involvement in surgical treatment decision making for breast cancer. Patient Educ Couns 2007, 65(3):387-395.

50. Zhang Y, Su H, Shang L, Li D, Wang R, Zhang R, Xu Y: Preferences and perceived involvement in treatment decision making among Chinese patients with chronic hepatitis. Med Decis Making 2011, 31(2):245-253.

51. Lantz PM, Janz NK, Fagerlin A, Schwartz K, Liu L, Lakhani I, Salem B, Katz SJ: Satisfaction with surgery outcomes and the decision process in a population-based sample of women with breast cancer. Health Services Res 2005, 40(3):745-767.

52. Pardon K, Deschepper R, Vander Stichele R, Bernheim J, Mortier F, Schallier D, Germonpre P, Galdermans D, Van Kerckhoven W, Deliens L: Are patients' preferences for information and participation in medical decision-making being met? Interview study with lung cancer patients. Palliat Med 2011, 25(1):62-70.

53. Davidson JR, Brundage MD, Feldman-Stewart D: Lung cancer treatment decisions: patients' desires for participation and information. Psychooncology 1999, 8(6):511-520.

54. Wallberg B, Michelson $H$, Nystedt M, Bolund C, Degner LF, Wilking N: Information needs and preferences for participation in treatment decisions among Swedish breast cancer patients. Acta Oncol 2000, 39(4):467-476

55. Jolicoeur LJA, O'Connor AM, Hopkins L, Graham ID: Women's decisionmaking needs related to treatment for recurrent ovarian cancer: a pilot study. Can Oncol Nurs J 2009, 19(3):117-121.

56. Mohamedali HZ, Breunis H, Panju A, Alibhai SMH: Information needs, decisional regret and satisfaction of older and younger adults with acute myeloid leukemia. J Geriatr Oncol 2010, 1(2):66-72.

57. Vogel BA, Leonhart R, Helmes AW: Communication matters: the impact of communication and participation in decision making on breast cancer patients' depression and quality of life. Patient Educ Couns 2009, 77(3):391-397.

58. Hawley ST, Janz NK, Hamilton A, Griggs JJ, Alderman AK, Mujahid M, Katz SJ: Latina patient perspectives about informed treatment decision making for breast cancer. Patient Educ Couns 2008, 73(2):363-370.
59. Davey HM, Lim J, Butow PN, Barratt AL, Redman S: Women's preferences for and views on decision-making for diagnostic tests. Soc Sci Med 2004 58(9):1699-1707.

60. Moreau A, Carol L, Dedianne MC, Dupraz C, Perdrix C, Laine X, Souweine G: What perceptions do patients have of decision making (DM)? Toward an integrative patient-centered care model. A qualitative study using focus-group interviews. Patient Educ Couns 2012, 87(2):206-211.

61. Sulmasy DP, Hughes MT, Thompson RE, Astrow AB, Terry PB, Kub J, Nolan MT: How would terminally ill patients have others make decisions for them in the event of decisional incapacity? A longitudinal study. J Am Geriatr Soc 2007, 55(12):1981-1988.

doi:10.1186/1472-6947-14-25

Cite this article as: Brom et al:: Congruence between patients' preferred and perceived participation in medical decision-making: a review of the literature. BMC Medical Informatics and Decision Making 2014 14:25.

\section{Submit your next manuscript to BioMed Central and take full advantage of:}

- Convenient online submission

- Thorough peer review

- No space constraints or color figure charges

- Immediate publication on acceptance

- Inclusion in PubMed, CAS, Scopus and Google Scholar

- Research which is freely available for redistribution
C) Biomed Central 